\title{
Artículos
}

\section{Conocimientos Disciplinarios y datos necesarios para la Eficiencia del agua en Edificios Verdes} \section{Buildings

\author{
Blandón Bautista, Enrique Ulises \\ (Enriqueulises29@gmail.com)
}

Disciplinary Knowledge and Data Needed for Water Efficiency in Green

\begin{abstract}
Water shortage has become one of the most serious issues in many countries of the world. It is crucial to implement initiatives to create awareness in the management and use of the potable water as well as to meet the water efficiency in green buildings.
\end{abstract}

The US green building guidelines LEED provide parameters or indicators relevant to the water performance in terms of quality and quantity. Architects and engineers of different disciplines require knowledge and data to successfully design and build sustainable buildings.

This research investigated knowledge disciplines and data needed in a project related to WE (water efficiency). It presented analyses comparing $\mathrm{WE}$ of green building projects, types and purposes of rainwater harvesting system, three discipline breakdown structures related to EA credits, and the academic courses related to EA.
Finally, the disciplines and data WE are explained by two case studies located in Taiwan (manufacturing Plant \& Cheng Kung Campus). The case studies about WE illustrated how to reduce the potable water consumption through the use of environmental-friendly strategies. This research process and results can help practitioners to gain a clear picture about how to design and implement a WE system for building projects.

Keywords

Sustainability, water efficiency, knowledge disciplines, water quantity, water use reduction, LEED

\section{Resumen}

La escasez de agua se ha convertido en uno de los problemas más graves en muchos países del mundo. Es crucial implementar iniciativas para crear conciencia en el manejo y uso del agua potable, así como para cumplir con el uso eficiente del agua en los edificios verdes.

Las directrices proporcionan los parámetros de la arquitectura sostenible LEED o indicadores relevantes para el 
funcionamiento del agua en términos de calidad y cantidad. Arquitectos e ingenieros de diferentes disciplinas requieren conocimientos y datos para diseñar y construir edificios sostenibles con éxito.

Se investigó disciplinas del conocimiento y los datos necesarios en un proyecto relacionado con EA (la eficiencia del agua). Presentó los análisis comparativos pasamos de proyectos de construcción verde, tipos y efectos del sistema de recogida de aguas pluviales, tres estructuras de desglose de la disciplina relacionadas con EA créditos y los cursos académicos relacionados con EA.

Por último, las disciplinas y los datos fueron explicados por dos casos de estudio ubicados en Taiwán (planta de fabricación y Campus Cheng Kung). Los estudios de caso sobre ilustramos cómo reducir el consumo de agua potable mediante el uso de estrategias amigables con el ambiente. Este proceso de investigación y los resultados pueden ayudar a los profesionales a obtener una idea clara acerca de cómo diseñar e implementar un sistema que para los proyectos de construcción.

\section{Palabras Claves}

Sostenibilidad, eficiencia hídrica, disciplinas del conocimiento, cantidad de agua, reducción del uso del agua, LEED

\section{Introducción}

Numerosas discusiones han tenido lugar durante las últimas tres décadas, produciendo contribución significativa al concepto de sostenibilidad. La conferencia de las Naciones Unidas (ONU) sobre el medio humano, celebrada en Estocolmo en 1972, fue importante porque por primera vez, se añadió el medio ambiente a la lista de los problemas globales.

Estimaciones recientes sitúan el monte de agua dulce necesaria para beber, la industria y el saneamiento, en torno al $2,5 \%$ del suministro total de agua MUNDO. Aproximadamente un tercio de esto es fácilmente accesible a los seres humanos a través de lagos, arroyos y ríos (Kubba, 2010). Con el recurso de agua dulce mundial contracción, hay una necesidad de reutilizar el agua (Deepika \& Pawan, 2010).

En los últimos años, algunos países han desarrollado la mayor parte de ellos, tienen la creación de conciencia en la conservación de este importante recurso a través de la creación de unas directrices para medir el uso del agua tales como: LEED (North America), BREAM (Great Britain), Green Star (Australia), CASBEE (Japan), DGNB (Germany), etc. (Seneviratne, 2007).

La eficiencia del agua (EA) es vital para dar cabida a los nuevos desarrollos, mientras que la protección de nuestros ríos, humedales, lagos y arroyos (Wise, 2010). Es importante para determinar el 
Arquitectura +

ISSN: 2518-2943

www.revistas.uni.edu.ni/index.php?journ

$\mathrm{al}=$ arquitectura
Vol. 1- Número 2 / Diciembre 2016

Conocimientos Disciplinarios y datos necesarios para la Eficiencia del agua en

Edificios Verdes/

Blandón Bautista, Enrique Ulises/

Enriqueulises29@gmail.com acceso y calcular las cantidades relativas EA o la conservación, acompañado de estrategias como el uso del agua, el funcionamiento y el rendimiento , la implementación, el ahorro de agua, etc. (Amy, 2001).

\section{Metodología}

Para desarrollar esta investigación, fue necesario revisar y considerar cuatro áreas principales. Estas áreas son: 1) la gestión del agua, 2) estrategias de agua, 3) sistema de calificación LEED - EA, y 4) cursos y disciplinas relacionadas con EA.

La revisión de la literatura relevante está enfocada en la eficiencia del agua (EA) y estrategias implementadas en los edificios verdes. La EA en LEED incluyen tres créditos: EA Paisajismo, tecnologías innovadoras de aguas residuales y la reducción del uso del agua.

Dos casos de estudios van a ser presentados, uno una planta de fabricación (EA) la aplicación y certificación), y el otro un campus universitario (aplicación del sistema de recolección de agua). En la planta de fabricación , se llevó a cabo un análisis para evaluar los créditos EA y qué datos son necesarios para el desarrollo del proyecto , donde obtuvo los más altos 5 puntos en la categoría de EA según LEED - V 2.2 .

El caso del Campus de Cheng Kung fue desarrollado con el objetivo de obtener la certificación como un campus

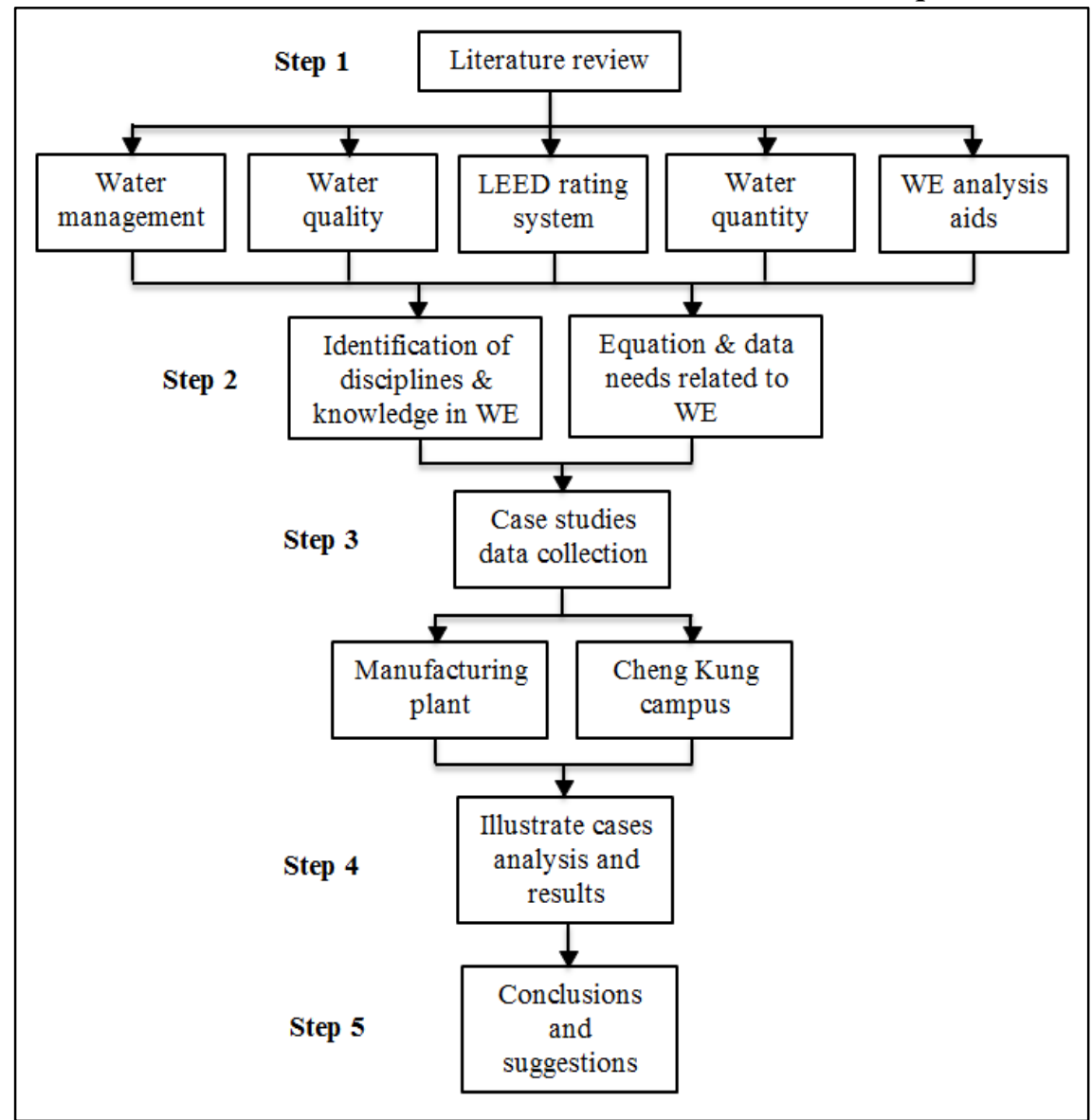

sostenible. Es necesario tener en cuenta otras categorías como la energía, el sitio del medio ambiente, la calidad ambiental interior , etc., para llamar a este caso un campus sostenible, de acuerdo con LEED. Estos no están en el alcance de esta investigación.

Esta investigación presenta EA a través de revisión de la literatura, análisis de crédito EA LEED, y estudios 
de casos. El proceso de investigación en la figura 1.1 consta de cinco pasos: (1) revisión de la literatura tales como la gestión del agua, calidad del agua, el sistema de calificación LEED, cantidad

\section{Desarrollo}

Manejo del Agua

La gestión del agua cubre una amplia gama de actividades, incluyendo el tratamiento de aguas residuales,
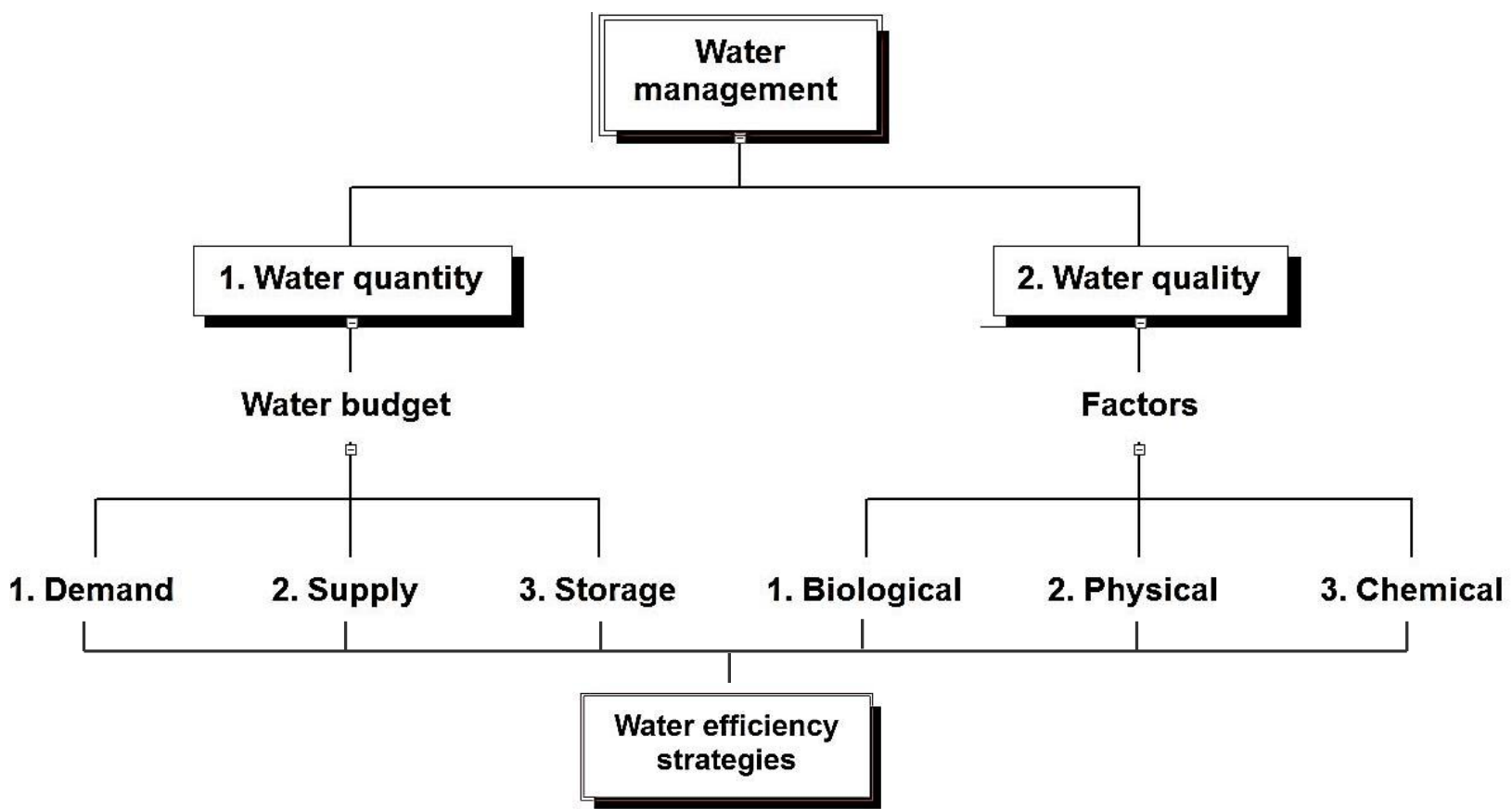

de agua y ayudas análisis de la eficiencia del agua. (2) Identificar las disciplinas del conocimiento, así como las ecuaciones y los datos relacionados con EA. (3) Recoger los datos relacionados con dos estudios de caso (la planta de fabricación y Campus Cheng Kung). (4) ilustran los cálculos de datos de casos, y los resultados del análisis. Por último, (5) presentan conclusiones y sugerencias. suministro de agua potable, y la regulación de las aguas superficiales (Ute \& Rainer, 2003). Además, la gestión del agua contribuye a preservar y proteger los recursos hídricos, centrándose en las condiciones de calidad y cantidad del agua. Sin embargo, es necesaria la creación de un presupuesto de agua, para calcular la cantidad de agua que se necesita para cubrir la demanda durante las estaciones secas (Zacharias y Dimitriou, 2003).

La siguiente figura muestra los componentes del manejo del agua que se resumen en una forma general. Estos 
componentes son la calidad y cantidad de agua, y sus respectivos subcomponentes. Por ejemplo, los factores de calidad del agua incluyen el factor biológico, físico y químico; la cantidad de agua discute presupuesto de agua y sus sub-componentes, tales como la demanda, suministro y almacenamiento.

The water quality factors are best broken down and analyzed in water quality section. It is also imperative to consider the stage of data collection, because it provides all the aspects needed to develop a right assessment and analysis for a project.

Además, estos componentes del manejo del agua son aplicables a EA estrategias, a través del análisis y cálculos, utilizando indicadores y otras herramientas (Zacharias y Dimitriou, 2003).

\section{Reciclaje y reutilización del Agua}

Debido al aumento de la demanda de agua en el sector industrial, se espera que el desarrollo tecnológico en los métodos de procesamiento y reutilización de agua para reducir la demanda de agua dulce. El reciclaje se define como el uso interno de las aguas residuales por el usuario original antes de la descarga a un sistema de tratamiento u otro punto de eliminación (Patel y Shah, 2008).

En las zonas pobladas bajas, tales como partes de América del Norte y Australia, las aguas grises (AG) reutilización son una práctica común debido a la escasez de agua, la creciente conciencia sobre la conservación del agua y la falta de plantas de tratamiento centralizado.

En los EE.UU, se estima que 1,7 millones de galones $(6,4$ millones de m3) por día de aguas residuales se reutilizan, y el uso de agua regenerada en base a volumen está creciendo a un $15 \%$ por año (EPA, 2004).

Los acontecimientos recientes en la tecnología y los cambios en las actitudes hacia la reutilización del agua sugieren que existe un potencial de reutilización GW en el mundo en desarrollo. GW representa la mayor fuente potencial de ahorro de agua en la residencia doméstica. Por ejemplo, la reutilización de GW interno para el riego de jardines se considera un avance significativo (Odeh y Jayyusi, 2003).

Los principales beneficios de la reutilización de aguas grises son que conserva los recursos de agua para usos potables, reduce la demanda de servicio en el suministro de agua potable (fuente , el tratamiento y la transmisión ) , reducir las exigencias a la infraestructura de aguas residuales aguas abajo (recogida , tratamiento y eliminación ), y podría ser un potencial de impactos sobre el medio receptor ( Mandal y Labhasetwar, 2011) .

Hoy en día , los ingenieros en la gestión del agua tienden a aplicar métodos que 
abordan sólo sub-partes para solucionar cualquier problema, mientras que los métodos de análisis de decisión multicriterio que combinan diferentes enfoques científicos en un paquete método para resolver el problema apenas se aplica ( Patel y Shah, 2009) . Esto significa que la ejecución del plan de gestión sostenible del agua puede proporcionar soluciones a diversos problemas relacionados con el agua, pero no es tarea fácil, ya que requiere un enfoque multidisciplinario y la lucha eficaz contra los numerosos problemas que puedan surgir.

Uno de los objetivos de un plan de gestión del agua es el presente y futuro de estimación de la demanda de agua, que debe ser considerado

cuidadosamente, ya que la mayoría determina la viabilidad del plan de gestión (Bithas, 2008).

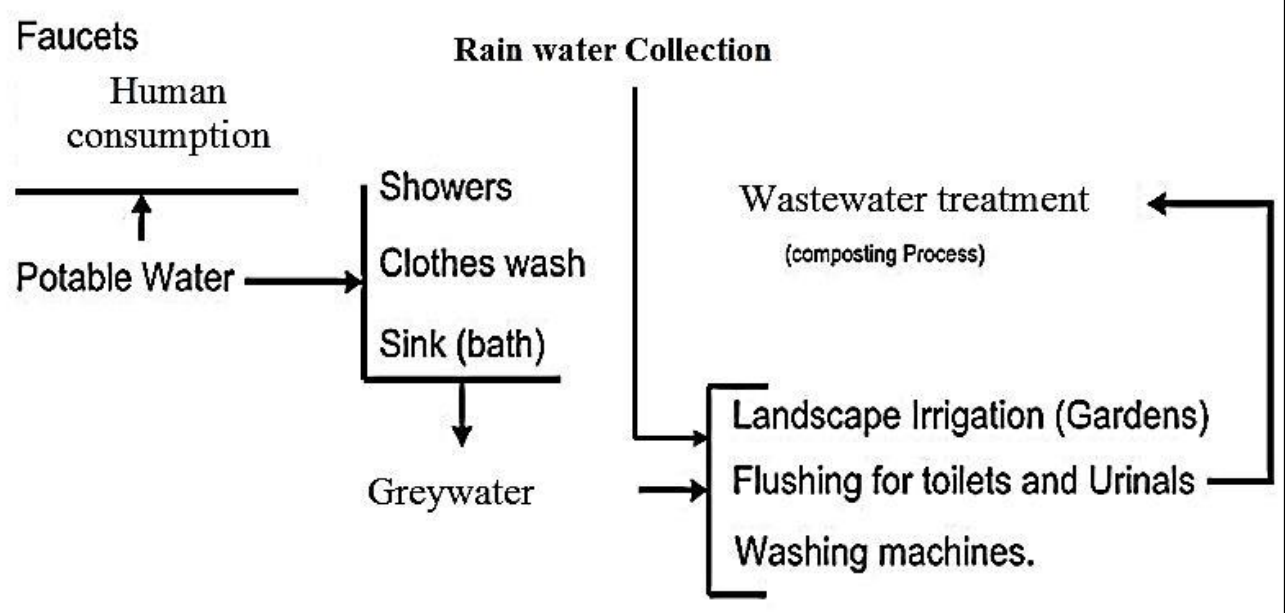

Otro de los objetivos en un plan de gestión del agua (WMP) es a una mayor preservación del medio ambiente sin comprometer el crecimiento económico (Bithas, 2008). Además de ahorrar agua de una organización requiere un plan, necesita incorporar un enfoque técnico y sistemático. Esta es la clave para lograr la conservación sostenible del agua dentro de una instalación (Seneviratne, 2007).
La siguiente figura ilustra un sistema de reciclado de agua. Este modelo se aplica sólo para las viviendas. Este diagrama muestra los componentes del sistema de reutilización del agua, tales como: recogida de aguas pluviales, agua potable, aguas grises y aguas residuales. Por ejemplo, el uso de agua potable está relacionada con actividades como el lavado de telas y beber, y la reutilización de aguas grises está relacionada con actividades como el riego de jardines, los inodoros y urinarios, etc. (USGBC, 2009). 
posición es la reducción de la escorrentía y la generación de carga, a través de controles de erosión, contaminación de origen y así sucesivamente, y la última posición es un tratamiento definitivo para los contaminantes y la retención para el riego, a través de franjas de vegetación, humedales de detención, y el estanque de retención para la reutilización de riego.
Aguas pluviales juega un papel importante en los estanques o humedales artificiales , cabe señalar que los flujos de estanques y lagos tienen que incluir la escorrentía de aguas pluviales y lixiviados de los sistemas sépticos fallidos, que pueden contener DBO , nutrientes, patógenos y trazas de constituyentes, tiene impacto en la calidad del agua (Metcalf \& Eddy, 2007).

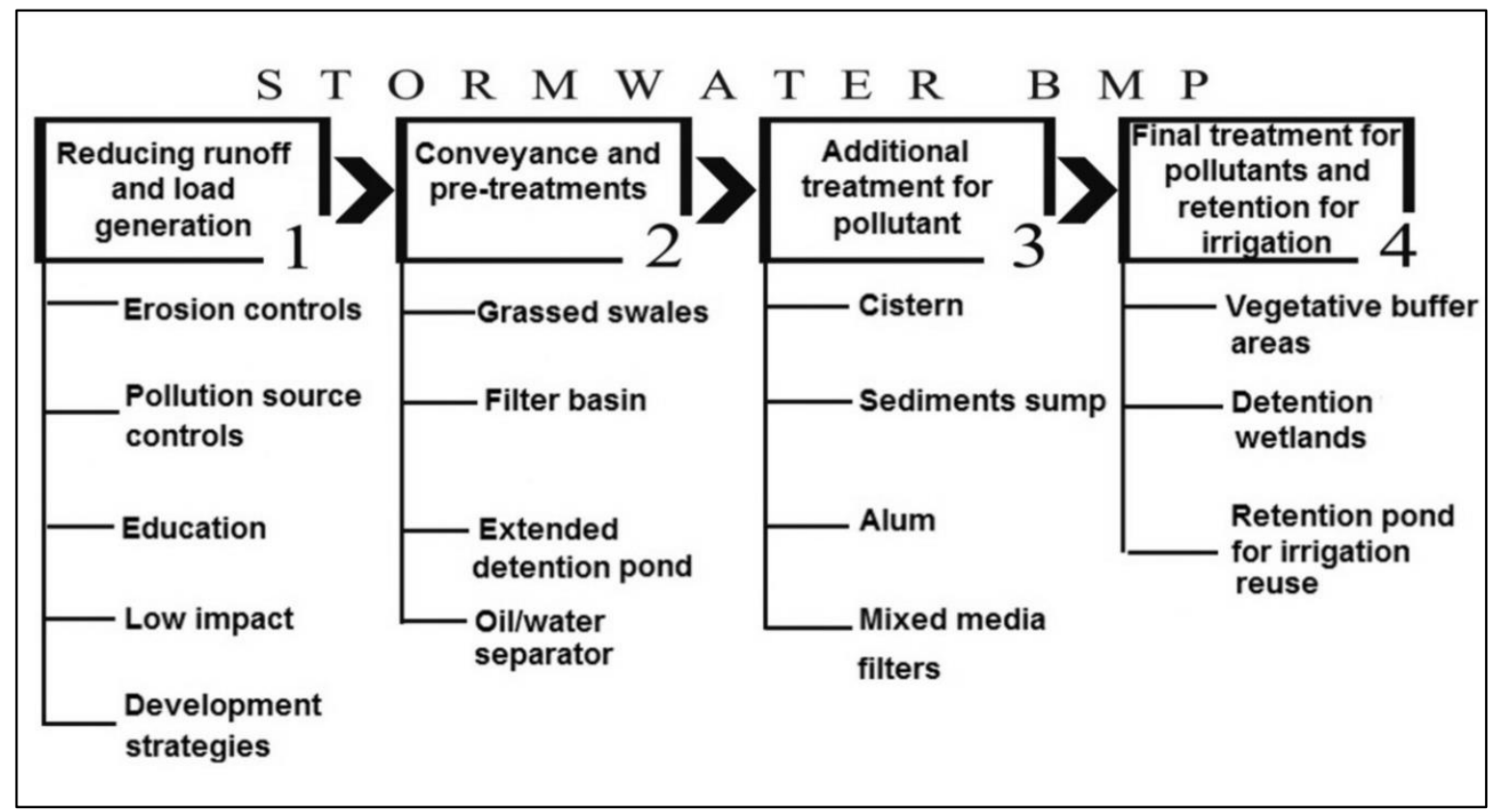

En los últimos años, ha habido un creciente interés en el uso de estrategias como jardinería con sistema de biofiltración , jardines de lluvia , células bio retención, estanques, que fomentan la infiltración de agua de lluvia para mitigar los impactos de agua subterránea y reducir el escurrimiento de contaminantes de las fuentes de agua como ríos , lago, lagunas ( Alejandro , 2006) .
Los impactos en la calidad del agua se originan a partir de fuentes antropogénicas y naturales, por ejemplo, el desarrollo humano en la cuenca tributaria puede resultar una mayor escorrentía de aguas pluviales que contiene fertilizantes y pesticidas. Las medidas para controlar las aguas pluviales han incluido las instalaciones de tratamiento de aguas pluviales, de captación de aguas pluviales y las 
cuencas de infiltración (Metcalf \& Eddy, 2007).

Sedimentos y desechos son algunos tipos de sólidos que se encuentran en las aguas pluviales y son generalmente la primera categoría de contaminantes para las aguas pluviales (Kayhanian, 2005). En adicional a los sedimentos y escombros, otros contaminantes de interés en las aguas pluviales son: nutrientes (nitrógeno y fósforo), metales pesados $(\mathrm{Cu}, \mathrm{Zn}$ y $\mathrm{Pb})$, bacterias y virus (animal contacto con el agua y los excrementos humanos), aceite y grasa hidrocarburos (coches e industriales), otros compuestos orgánicos (disolventes, limpiadores y selladores), pesticidas y sales (cloruros de sodio y calcio) (Ronny \& Robert, 2009).

\section{Calidad del Agua}

En todo el mundo, los contaminantes procedentes de las actividades humanas han degradado seriamente la calidad del agua. Gestores de la calidad del agua están tratando con el control de la contaminación que los seres humanos están emitiendo, para asegurar que el agua puede ser adecuado para sus usos previstos y las generaciones futuras (Davis y Masten, 2004).

La calidad del agua es un término usado para describir la físicas, químicas y biológicas de agua con respecto a su idoneidad para un uso particular (Lin, 2007). La autoridad de evaluación de la calidad del agua (WQAA) se ha establecido recientemente para coordinar de manera efectiva y mejorar el trabajo de monitoreo de la calidad del agua por varias organizaciones en los Estados Unidos (Patel y Shah, 2009).

Ofertas de gestión de la calidad del agua en primer lugar con los principales tipos de contaminantes del agua y sus fuentes, una amplia gama de contaminantes vertidos a las aguas superficies se pueden agrupar en grandes categorías. Las aguas residuales domésticas y los desechos industriales se denominan fuentes puntuales porque en general se recogieron mediante una red de tuberías o canales y se transportan a un solo punto de descarga en las aguas receptoras. En general, la contaminación de fuentes puntuales puede ser reducido o eliminado a través de la minimización de residuos y tratamiento de aguas residuales adecuado antes de su descarga a un cuerpo natural de agua como ríos, lagos, lagunas y etc (Davis y Masten, 2004).

La siguiente figura ilustra los diferentes factores que afectan directamente a la calidad del agua, tales como, biológicos, físicos y químicos (Ronny \& Carrow, 2009). Los factores sección biológicos tales como patógenos humanos, patógenos de las plantas y así sucesivamente. Los factores físicos son aquellos que un color de fondo, la 


\section{Arquitectura +}

ISSN: 2518-2943

www.revistas.uni.edu.ni/index.php?journ

$\mathrm{al}=$ arquitectura
Vol. 1- Número 2 / Diciembre 2016

Conocimientos Disciplinarios y datos necesarios para la Eficiencia del agua en

Edificios Verdes/

Blandón Bautista, Enrique Ulises/

Enriqueulises29@gmail.com

turbidez, el olor y el total de sólidos en suspensión.

Finalmente, los factores químicos es tal vez uno de los más importantes en la gestión de la calidad del agua, incluyendo factores tales como el oxígeno disuelto, orgánicos biodegradables (DBO, DQO y COT), surfactantes, pesticidas, grasa, aceite y así sucesivamente. Existentes problemas de escasez y calidad del agua experimentaron prácticamente en todo el mundo a hacer la recolección de agua es un tema crítico para el desarrollo sostenible (Patel y Shah, 2008). 
Arquitectura +

ISSN: 2518-2943

www.revistas.uni.edu.ni/index.php?journ

$\mathrm{al}=$ arquitectura
Vol. 1- Número 2 / Diciembre 2016

Conocimientos Disciplinarios y datos necesarios para la Eficiencia del agua en

Edificios Verdes/

Blandón Bautista, Enrique Ulises/ Enriqueulises29@gmail.com
La calidad del agua está directamente relacionada con la calidad del agua de lluvia, la calidad de las aguas residuales y la calidad de las aguas pluviales, y las consumo humano o el medio ambiente, como el riego en el paisaje, potable y el consumo de agua no potable, y aseo $\mathrm{y}$ accesorios urinarios.

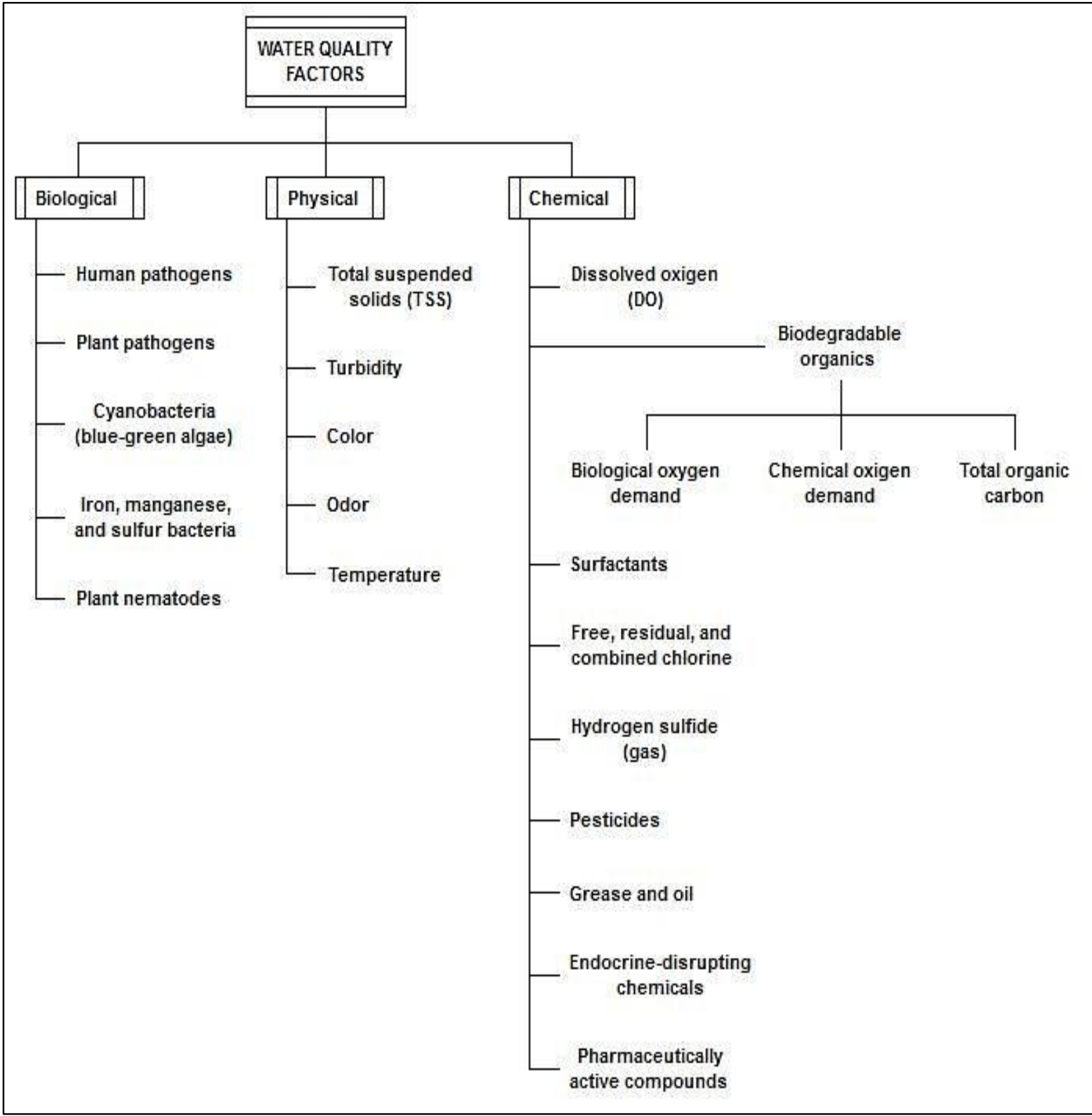

tesis de agua se puede reutilizar o

Disciplinas relacionadas con la eficiencia del reciclar para diferentes propósitos o agua actividades, relacionadas con el 


\begin{tabular}{|c|c|c|}
\hline $\begin{array}{l}\text { Arquitectura + } \\
\text { ISSN: } 2518-2943 \\
\text { www.revistas.uni.edu.ni/index.php?journ } \\
\text { al=arquitectura }\end{array}$ & & $\begin{array}{r}\text { Vol. 1- Número 2 / Diciembre } 2016 \\
\text { Conocimientos Disciplinarios y datos } \\
\text { necesarios para la Eficiencia del agua en } \\
\text { Edificios Verdes/ } \\
\text { Blandón Bautista, Enrique Ulises/ } \\
\text { Enriqueulises29@gmail.com }\end{array}$ \\
\hline
\end{tabular}

Las estructuras de desglose de EA disciplinas relacionadas se presentan del propietario para los participantes del proyecto. Una ventaja importante es que las sinergias a menudo se logran cuando las distintas partes contribuyen con su asesoramiento y experiencia en las primeras fases del diseño, que les permite realizar sus propias funciones que se mejora el conocimiento del proyecto en su totalidad. En los casos de recogida de agua de lluvia y sistemas de tratamiento de aguas residuales que implican múltiples disciplinas del proyecto, por lo que es particularmente importante para articular claramente las responsabilidades (Kubba, 2010).

Para desarrollar estas estructuras disciplinas para cada crédito, era necesario profundizar en cada uno de crédito y encontrar los datos relevantes al tema y asignarlos a diferentes disciplinas, por ejemplo, los datos relativos a la calidad del agua está más cerca de o relacionados con disciplinas como la ingeniería ambiental e ingeniero químico y así sucesivamente.

A pesar de la falta de un entendimiento común de lo que la sostenibilidad es y las diversas interpretaciones entre diferentes disciplinas, existe un entendimiento general de que todo un sistema, se necesita visión a largo plazo para evaluar y sostenibilidad enfoque, particularmente en el caso de la gestión de los recursos hídricos. También es importante establecer una mejor comprensión de la terminología relacionada con la conservación del agua para facilitar un mejor trabajo entre diferentes disciplinas (Metcalf y Eddy, 2007)

Baste decir aquí que esto es precisamente por qué se necesita un proceso de diseño integrado, desde el comienzo de la conceptualización del proyecto hasta la construcción, con la participación de todas las disciplinas relacionadas en un proyecto típico y trabajando juntos, incluyendo el arquitecto, ingeniero, contratista, propietario, proveedores, y otros (LEED, 2009).

En las siguientes figuras 2 a 3,4 estructuras actuales de disciplinas y sus respectivos datos y conocimientos para desarrollar un proyecto de uso eficiente del agua y lograr todos sus respectivos créditos. Estas cifras se dividen en tres secciones que representan los créditos EA. 


\section{Arquitectura +}

ISSN: 2518-2943

www.revistas.uni.edu.ni/index.php?journ

al=arquitectura
Vol. 1- Número 2 / Diciembre 2016

Conocimientos Disciplinarios y datos necesarios para la Eficiencia del agua en Edificios Verdes/

Blandón Bautista, Enrique Ulises/ Enriqueulises29@gmail.com

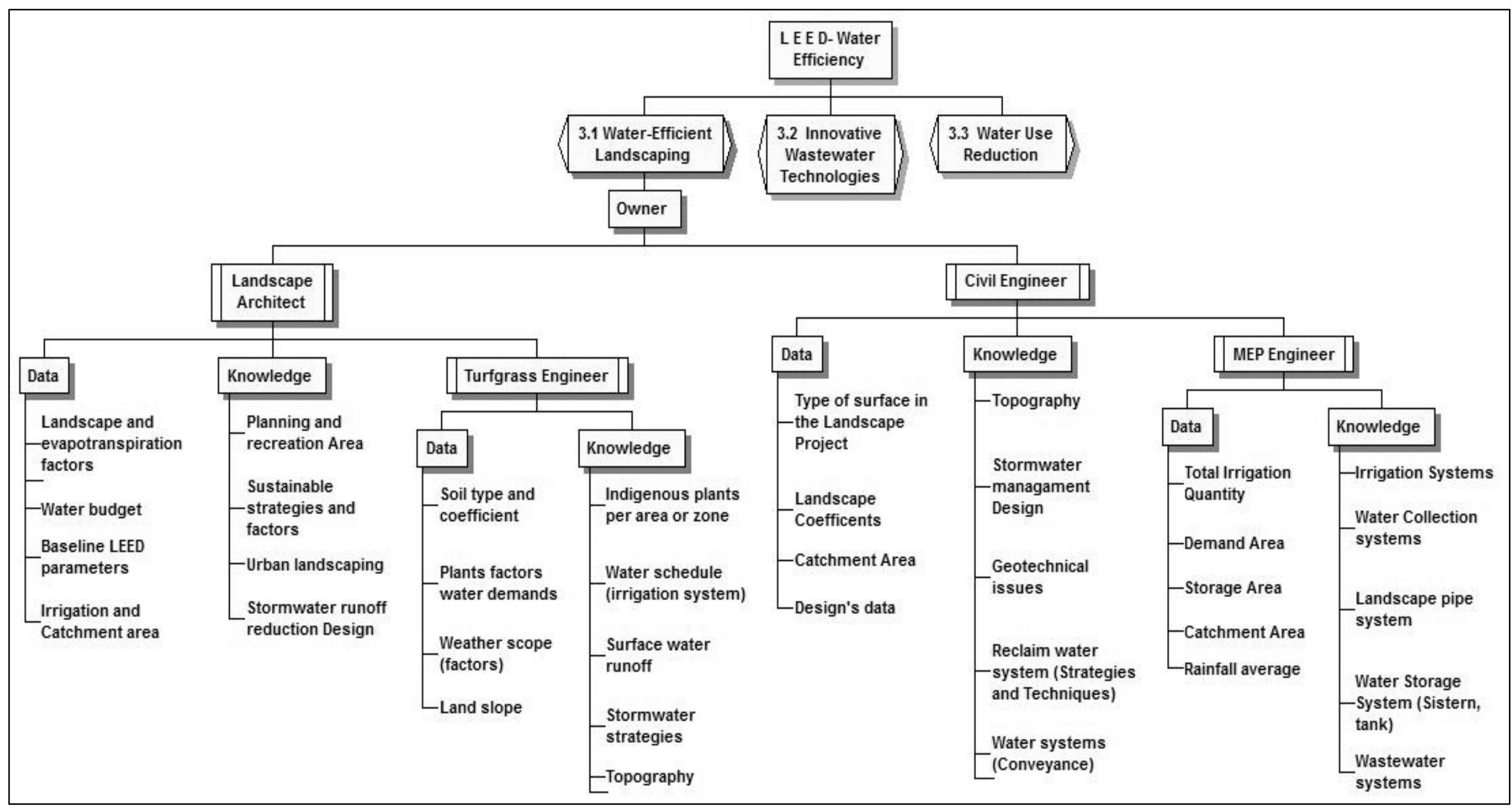




\begin{tabular}{l|r}
$\begin{array}{l}\text { Arquitectura + } \\
\text { ISSN: } 2518-2943 \\
\text { www.revistas.uni.edu.ni/index.php?journ } \\
\text { al=arquitectura }\end{array}$ & $\begin{array}{r}\text { Vol. 1- Número 2/ Diciembre 2016 } \\
\text { Conocimientos Disciplinarios y datos } \\
\text { necesarios para la Eficiencia del agua en } \\
\text { Edificios Verdes/ }\end{array}$ \\
\end{tabular}

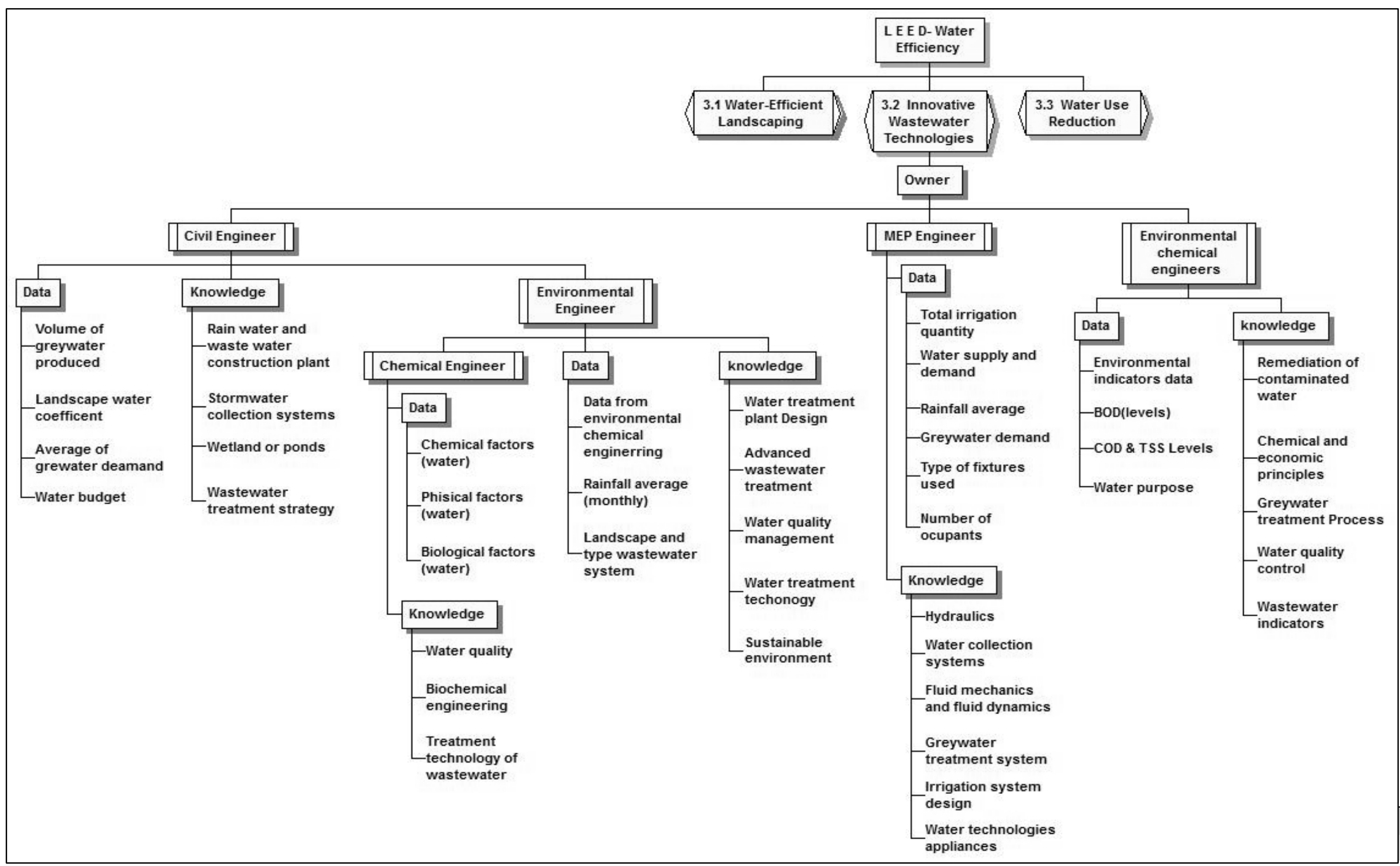




\begin{tabular}{l|r}
$\begin{array}{l}\text { Arquitectura + } \\
\text { ISSN: 2518-2943 } \\
\text { www.revistas.uni.edu.ni/index.php?journ } \\
\text { al=arquitectura }\end{array}$ & $\begin{array}{r}\text { Vol. 1- Número 2/ Diciembre 2016 } \\
\text { Conocimientos Disciplinarios y datos } \\
\text { necesarios para la Eficiencia del agua en } \\
\text { Edificios Verdes/ }\end{array}$ \\
Blandón Bautista, Enrique Ulises/ \\
Enriqueulises29@gmail.com
\end{tabular}

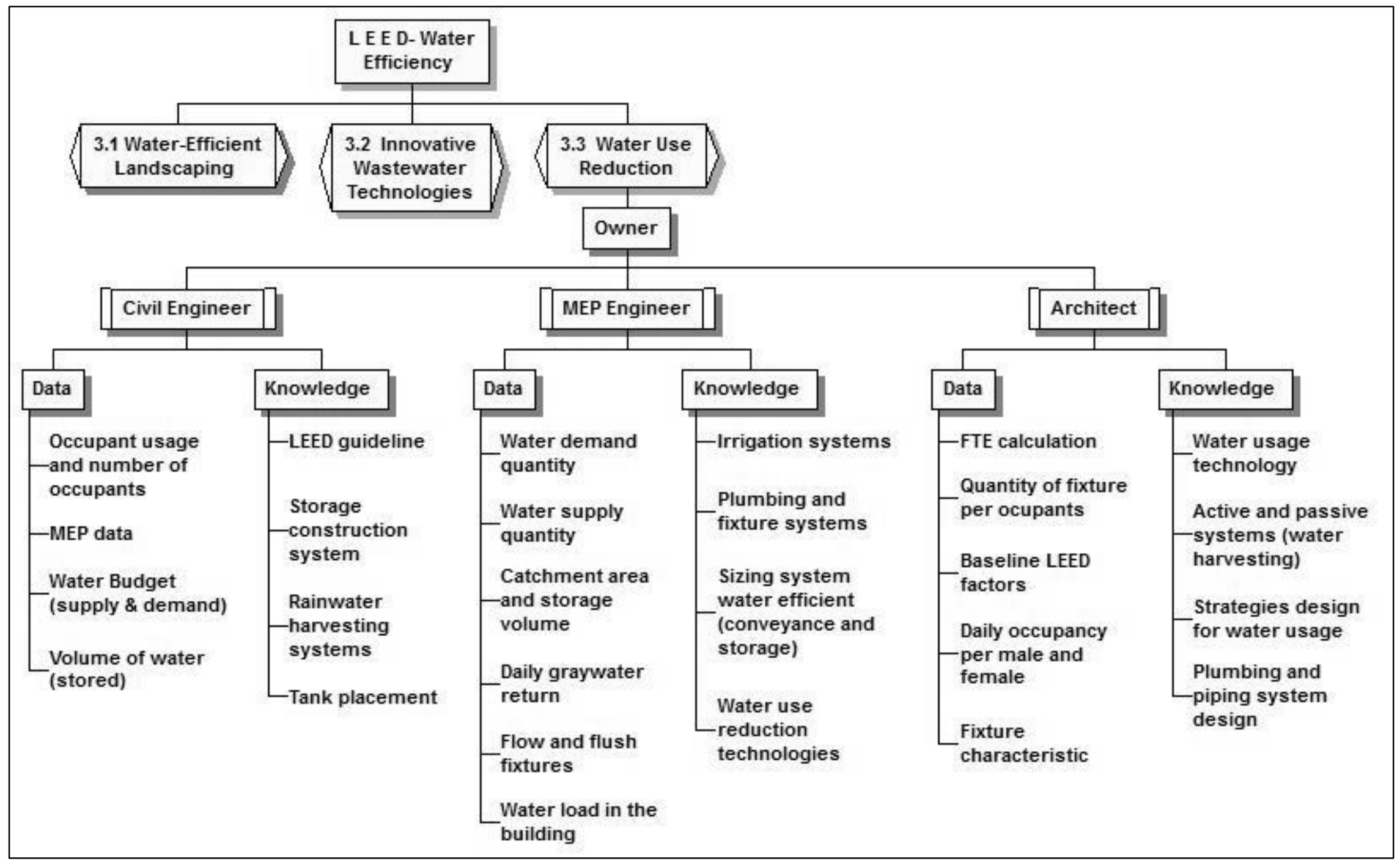




Arquitectura +
ISSN: 2518-2943
www.revistas.uni.edu.ni/index.php?journ
al=arquitectura

Arquitectura +

www.revistas.uni.edu.ni/index.php?journ

$\mathrm{al}=$ arquitectura
Vol. 1- Número 2 / Diciembre 2016

Conocimientos Disciplinarios y datos necesarios para la Eficiencia del agua en Edificios Verdes/

Blandón Bautista, Enrique Ulises/ Enriqueulises29@gmail.com

\section{Conclusiones}

Esta investigación ha presentado disciplinas del conocimiento y los datos relacionados con la EA en los edificios verdes. Se revisó y se analizó el sistema de calificación LEED en la categoría de EA para reducir el agua potable en edificios a través de la utilización de los datos e indicadores. A continuación, dos estudios de caso fueron presentados: una planta de fabricación y un campus universitario. En este capítulo se presentan las conclusiones y sugerencias para futuras investigaciones.

1. Una contribución de esta investigación se encuentra en áreas de gestión del agua, a través del uso de figuras, tablas, ecuaciones, datos y disciplinas de conocimiento necesarios para desarrollar un proyecto de EA. También ilustra qué tipo de estrategias están más relacionados con los créditos en la categoría de LEED-EA.

2. En esta investigación, las disciplinas de conocimiento que están directamente vinculados con los proyectos de EA son: medio ambiente, civil, química, mecánica, y los ingenieros hidráulicos, y el arquitecto (paisajismo). Disciplinas más relevantes son los ingenieros ambientales $\mathrm{y}$ civiles, $\mathrm{y}$ el arquitecto.

3. De acuerdo con la evaluación desarrollada en esta investigación, las estrategias eficaces son las que implantan sistemas activos (agua de lluvia y recolección de aguas grises), y las estrategias se utilizan principalmente en actividades como el riego.

4. Esta investigación ayuda a los profesionales o estudiantes que tienen una idea clara acerca de cómo diseñar un sistema de EA mediante la identificación de los datos relacionados con las características de sus proyectos y disciplinas mediante el uso de tablas, figuras y ecuaciones.

5. Esta investigación presenta los datos y ecuaciones necesarias para aplicar en las zonas rurales, donde se complica el acceso al agua potable. Además, la aplicación de esta investigación en otros países es posible porque los factores, coeficientes y otros parámetros pueden ser fácilmente de entrada en las ecuaciones.

6. En el caso de estudio, la planta de fabricación presenta un complejo sistema de tanques de recogida de aguas pluviales. El agua se utiliza para diferentes fines, como la irrigación y al ras en el inodoro y urinarios. Al mismo tiempo que innovar con el agua capturada de agua condensada (MAU), integrado al sistema de aguas grises de recuperación. En este caso la planta invirtió más en la tecnología de aguas residuales innovador que otros sistemas, ya que procesa el agua de recuperación y el agua en general reutilización en $40 \%$ y $13 \%$, respectivamente. Y sólo el 1,7\% y el 0,3\% a partir de MAU agua condensada y el agua de lluvia, respectivamente, es para propósitos de irrigación.

7. En el caso de estudio Campus Chen Kung, el resultado del análisis del sistema tiene un promedio de 483.697 galones / mes (1.830.992 l / meses) en el área de la demanda que la inclusión de sólo el riego de jardines hasta el momento. El área de suministro (área de influencia) ofrece un promedio de mes de 2, 273,144 galones / mes (8.604.786 l / mes).

8. El área de almacenamiento tiene una capacidad de 3,935m3 de acuerdo con el 


Arquitectura +
ISSN: 2518-2943
www.revistas.uni.edu.ni/index.php?journ
al=arquitectura

Vol. 1- Número 2 / Diciembre 2016

Conocimientos Disciplinarios y datos necesarios para la Eficiencia del agua en Edificios Verdes/

Blandón Bautista, Enrique Ulises/ Enriqueulises29@gmail.com programa de agua viva campus. Se cubre parte del volumen de almacenamiento de agua para utilizar cisternas situadas en cada edificio y el uso de la filtración anterior antes de llegar al estanque o vía acuática.

9. La demanda de aguas grises en Cheng Kung Campus por mes (inodoros y urinarios) utilizando un sistema de accesorios de eficiencia es de alrededor de 462.790 galones / mes (1.751.850 l/ meses). Cabe destacar que este sistema solo EA suministra la demanda de agua para el riego de jardines y el uso de agua no potable, como inodoros y urinarios.

\section{Recomendaciones:}

Con base en los hallazgos de esta investigación, se sugiere lo siguiente.

1. Reunir más datos relativos a la construcción ecológica ya certificado por LEED en Taiwán en relación con EA. Esto daría una idea clara acerca de cada crédito en EA y cómo esta categoría interactúa con otras categorías o créditos como categoría SS con créditos como la calidad y cantidad de las aguas pluviales.

2. El ámbito de investigación es grande por lo que algunos aspectos no fueron muy estudiados de forma profundamente. La investigación futura puede seleccionar algunos sub-temas de esta investigación para explorar más a fondo, sobre todo en las disciplinas de conocimiento e interrelación de datos.

3. En el caso de la planta de fabricación, tal vez en el futuro, integrar en el sistema de recuperación de agua con agua procedente de los chips de decisiones.

4. En el caso del Campus Chen Kung, es necesario integrar más estrategias en términos de escorrentía de aguas pluviales y desarrollar un estudio de impacto ambiental en el campus, con el fin de utilizar factores $o$ coeficiente mayor precisión.

En relación con la escorrentía de aguas pluviales a causa del grado de contaminación de esta agua, si es que va a ser considerados como agua recuperada para la recogida Campus sistema de Cheng Kung, debe ser separado del sistema de recolección de agua de lluvia.

\section{Bibliografías y Referencias}

1. Abdulla, F. A., \& Al-Shareef, A. W. (2009). Roof rainwater harvesting systems for household water supply in Jordan. Desalination, 243(1-3), 195-207.

2. Al-Jayyousi, O. R. (2003). Greywater reuse: towards sustainable water management. Desalination, 156(1-3), 181-192.

3. Adriene LaBranche, Hans-Otto Wack, Ph.D., David Crawford, 2007. Virginia Rainwater Harvesting Manual. Compiled by The Cabell Brand Center, Aug.

4. Alexander Hamilton (2010). Urban Green Council N.Y. Published by USGBC. Proposal developed by the Water Efficiency \& Building Stormwater Committee.

5. Azhar, S., Carlton, W. A., Olsen, D., \& Ahmad, I. (2011). Building information modeling for sustainable design and LEED ${ }^{\circledR}$ rating analysis. Automation in Construction, 20(2), 217-224.

6. Berger, M., \& Finkbeiner, M. (2010). Water Foot printing: How to Address Water Use in Life Cycle Assessment? Sustainability, 2(4), 919-944.

7. Bithas, K. (2008). The sustainable residential water use: Sustainability, efficiency and social equity. The European experience. Ecological Economics, 68(1-2), 221-229.

8. Bradshaw, Vickers. (1993). Building Control Systems (2nd edition.). Wiley. 


Arquitectura +
ISSN: 2518-2943
www.revistas.uni.edu.ni/index.php?journ
al=arquitectura

Vol. 1- Número 2 / Diciembre 2016

Conocimientos Disciplinarios y datos necesarios para la Eficiencia del agua en Edificios Verdes/

Blandón Bautista, Enrique Ulises/ Enriqueulises29@gmail.com
9. Burkhard, R., Deletic, A., \& Craig, A. (2000). Techniques for water and wastewater management: a review of techniques and their integration in planning. Urban Water, 2(3), 197-221.

10. Carrow, R. N., Duncan, R. R., \& Huck, M. T. (2008). Turfgrass and Landscape Irrigation Water Quality: Assessment and Management (1st ed.). CRC Press.

11. Carter, T., \& Keeler, A. (2008). Life-cycle cost-benefit analysis of extensive vegetated roof systems. Journal of Environmental Management, 87(3), 350-363.

12. Chang, A. S. (2009).Campus Living Water Program. Banyan Research Express, NCKU. Taiwan.

13. Company, M. \& E., Inc. an AECOM, Asano, T., Burton, F., Leverenz, H., Tsuchihashi, R., \& Tchobanoglous, G. (2007). Water Reuse: Issues, Technologies, and Applications (1st edition.). McGraw-Hill

14. Czemiel Berndtsson, J. (2010). Green roof performance towards management of runoff water quantity and quality: A review. Ecological Engineering, 36(4), 351-360.

15. Davis, A. P., \& McCuen, R. H. (2010). Stormwater Management for Smart Growth (1st ed. Softcover of orig. ed. 2005 edition.). Springer.

16. Davis, M. (2010). Water and Wastewater Engineering (1st edition.). McGraw-Hill Professional.

17. Davis, M., \& Masten, S. (2008). Principles of Environmental Engineering \& Science (2nd edition.).

McGraw-Hill Science/Engineering/Math.

18. Dean, A. (2003). Green by Design: Creating a Home for Sustainable Living (1st edition.). Gibbs Smith.

19. Peot M. \& Schumacher B (2009). LEED BD\&C Practice Exam: Building Design \& Construction. Professional Publications, Inc.

20. Fan, J \& Fan, W. (2006). Treatment and reuse of toilet wastewater by an airlift external circulation membrane bioreactor. Process Biochemistry, 41(6), 1364-1370.
21. Friend, H. D., \& Coutts, S. S. (2006). Achieving sustainable recycled water initiatives through public participation. Desalination, 187(1-3), 159-166.

22. Ghisi, E. (2006). Potential for potable water savings by using rainwater in the residential sector of Brazil. Building and Environment, 41(11), 1544-1550.

23. Ilha, M., Oliveira, L., \& Gonçalves, O. (2009). Environmental assessment of residential buildings with an emphasis on water conservation. Building Services Engineering Research and Technology, 30(1), 15 -26.

24. I. Zabalza, A. Aranda, S. Scarpellini, S. Díaz, (2009). Life Cycle Assessment in Building Sector: State of the Art and Assessment of Environmental Impact for Building Materials. CIRCE - Zaragoza, Spain.

25. Kenney, D. S. (2006). In Search of Sustainable Water Management: International Lessons for the American West and Beyond. Edward Elgar Publishing.

26. Kinkade-Levario, H. (2007). Design for Water: Rainwater Harvesting, Stormwater Catchment, and Alternate Water Reuse (1st edition.). New Society Publishers.

27. Krygiel, E., \& Nies, B. (2008). Green BIM: Successful Sustainable Design with Building Information Modeling (1st edition.). Sybex.

28. Kubba, S. (2009). LEED Practices, Certification, and Accreditation Handbook. Butterworth-Heinemann.

29. Lambooy, T. (2011). Corporate social responsibility: sustainable water use. Journal of Cleaner Production, 19(8), 852-866.

30. LEED 2.2, 2005. New Construction \& Major Renovations. USGBC, for public use and display, Version 2.2. Oct.

31. Lin, S., \& Lee, C. (2007). Water and Wastewater Calculations Manual, 2nd Ed. (2nd edition.). McGraw-Hill Professional.

32. Lundin, M., \& Morrison, G. M. (2002). A life cycle assessment based procedure for development of environmental sustainability indicators for urban water systems. Urban Water, 4(2), 145-152. 


Arquitectura +
ISSN: 2518-2943
www.revistas.uni.edu.ni/index.php?journ
al=arquitectura

Vol. 1- Número 2 / Diciembre 2016

Conocimientos Disciplinarios y datos necesarios para la Eficiencia del agua en Edificios Verdes/

Blandón Bautista, Enrique Ulises/ Enriqueulises29@gmail.com
33. Mandal, D., Labhasetwar, P., Dhone, S., Dubey, A. S., Shinde, G., \& Wate, S. (2011). Water conservation due to Greywater treatment and reuse in urban setting with specific context to developing countries. Resources, Conservation and Recycling, 55(3), 356-361.

34. Mihelcic, J. R., \& Zimmerman, J. B. (2009). Environmental Engineering: Fundamentals, Sustainability, Design (1st edition.). Wiley.

35. Palme, U., \& Tillman, A.-M. (2008). Sustainable development indicators: how are they used in Swedish water utilities? Journal of Cleaner Production, 16(13), 1346-1357.

36. Patricia H. Waterfall, 2004. Harvesting Rainwater for Landscape Use. Second Edition, University of Arizona, Tucson.

37. Patel, A. S., \& Shah, D. L. (2009). Water Management: Conservation, Harvesting and Artificial Recharge. To New Age International Pvt Ltd Publishers.

38. Russ, T. (2009). Site Planning and Design Handbook, Second Edition (2nd ed.). McGraw-Hill Professional.

39. Robert E. 2008. Sustainable Design Through BIM and Analysis. Middlebrooks, AIA.

40. Scheuer, C., Keoleian, G. A., \& Reppe, P. (2003). Life cycle energy and environmental performance of a new university building: modeling challenges and design implications. Energy and Buildings, 35(10), 1049-1064.

41. Seneviratne, M. (2007). A Practical Approach to Water Conservation for Commercial and Industrial Facilities. Elsevier Science.

42. Simon, U., Brüggemann, R., \& Pudenz, S. (2004). Aspects of decision support in water management--example Berlin and Potsdam (Germany) I--spatially differentiated evaluation. Water Research, 38(7), 18091816.
43. USGBC, 2009). LEED Reference Guide for Green Building Design and Construction (2009th edition.). US. Green Building Council.

44. US. EPA, (2004). Guidelines for Water Reuse. Municipal Support Division. Washington, DC.

45. Vickers, A. (2001). Handbook of Water Use and Conservation: Homes, Landscapes, Industries, Businesses, Farms (1st edition.). Water Plow Press.

46. Villarreal, E. L., \& Dixon, A. (2005). Analysis of a rainwater collection system for domestic water supply in Ringdansen, Norrköping, Sweden. Building and Environment, 40(9), 1174-1184.

47. Wang, B. J. (2005). Optimization of Semiconductor Processing Water Management Strategy. Journal of Environmental Informatics, 5(2), 81-88. doi:10.3808/jei.200500049

48. Williams, D. R. (1993). EPAct: The Energy Policy Act of 1992: the reference addition. AC, Inc. Press.

49. Wise, A. F. E., \& Swaffield, J. (2002). Water, Sanitary and Waste Services for Buildings, Fifth Edition (5th edition.). ButterworthHeinemann.

50. Yudelson, J. (2007). Green Building A to Z: Understanding the Language of Green Building. New Society Publishers.

51. Zacharias, I., Dimitriou, E., \& Koussouris, T. (2003). Developing sustainable water management scenarios by using thorough hydrologic analysis and environmental criteria. Journal of Environmental Management, 69(4), 401-412.

52. Jack Holmgreen, 2010. Marine Engineer. http://EzineArticles.com/4848897 